\title{
Transverse mixing of ellipsoidal particles in a rotating drum
}

\author{
Siyuan $\mathrm{He}^{1}$, Jieqing Gan ${ }^{1}$, David Pinson ${ }^{2}$, and Zongyan Zhou ${ }^{1, *}$ \\ ${ }^{1}$ Department of Chemical Engineering, Monash University, Melbourne, VIC 3800, Australia \\ ${ }^{2}$ BlueScope Steel, Port Kembla, NSW 2505, Australia
}

\begin{abstract}
Rotating drums are widely used in industry for mixing, milling, coating and drying processes. In the past decades, mixing of granular materials in rotating drums has been extensively investigated, but most of the studies are based on spherical particles. Particle shape has an influence on the flow behaviour and thus mixing behaviour, though the shape effect has as-yet received limited study. In this work, discrete element method (DEM) is employed to study the transverse mixing of ellipsoidal particles in a rotating drum. The effects of aspect ratio and rotating speed on mixing quality and mixing rate are investigated. The results show that mixing index increases exponentially with time for both spheres and ellipsoids. Particles with various aspect ratios are able to reach well-mixed states after sufficient revolutions in the rolling or cascading regime. Ellipsoids show higher mixing rate when rotational speed is set between 25 and $40 \mathrm{rpm}$. The relationship between mixing rate and aspect ratio of ellipsoids is established, demonstrating that, particles with aspect ratios of 0.5 and 2.0 achieve the highest mixing rates. Increasing rotating speed from $15 \mathrm{rpm}$ to $40 \mathrm{rpm}$ does not necessarily increase the mixing speed of spheres, while monotonous increase is observed for ellipsoids.
\end{abstract}

\section{Introduction}

Rotating drums are widely used in industry for granular mixing. The mixing process of spherical particles has been studied extensively in the past decades. For example, Henein et al. [1] studied the segregation in a rotary kiln experimentally. Alizadeh et al. [2] studied the mixing and segregation process in a rotating drum by particle tracking method. Liu et al [3] used Discrete Element Method (DEM) to simulate the transverse mixing of wet particles. They found that the mixing of dry particles was poorest at $64 \%$ filling level and increasing the cohesion at that level improved particle mixing. Similarly, Arntz et al [4] focused on the impact of rotational speed and filling level by DEM. They found that there was strong correlation between flow regime and segregation pattern and the most intense segregation occurred when the fill fraction is larger than $65 \%$.

Though comprehensive study have been implemented on mixing of spherical particles, the mixing of non-spherical particles have received limited study yet. Particle shape is an important factor that affects the mixing behaviour and thus mixing rate. Geng et al [5] focused on the mixing rate of cylinder-shaped particles and found that the mixing rate of slender particles was faster than for spheres. Pereira et al [6] studied the streak patterns formation in binary granular mixture, considering both the size and shape effects. They proposed that the growth and stabilisation of a regular streak pattern required the two sets of particles to differ significantly in angle of repose. Though the efforts have been made on the mixing of non-spherical particles, e.g. slender particles or super-quadrics, the mixing process of ellipsoidal particles with different aspect ratios has not been studied yet. In this work, we focus on the mixing of ellipsoids with various aspect ratios in comparison with spheres. The mixing patterns for both ellipsoids and spheres are presented to examine the evolution of the structure of the beds. By introducing mixing index and mixing rate, the quality and speed of mixing process are quantified, respectively.

\section{Simulation methods and conditions}

According to the DEM proposed by Cundall and Strack [7], a particle can have two types of motion: translational and rotational, which are determined by Newton's second law of motion. DEM for ellipsoidal particles have been reported by Dziugys [8]. The DEM for ellipsoids was developed by the authors. The governing equations for the translational and rotational motion of particle $i$ with radius $\mathrm{R}_{\mathrm{i}}$, mass $\mathrm{m}_{\mathrm{i}}$, and moment of inertia $\mathrm{I}_{\mathrm{i}}$ can be written as:

and

$$
m_{i} \frac{d \mathbf{v}_{i}}{d t}=\sum_{j=1}^{k_{c}}\left(\mathbf{f}_{c, i j}+\mathbf{f}_{d, i j}+\mathbf{f}_{v, i j}\right)+m_{i} \mathbf{g}
$$

$$
I_{i} \frac{d \boldsymbol{\omega}_{i}}{d t}=\sum_{j=1}^{k_{c}}\left(\mathbf{M}_{t, i j}+\mathbf{M}_{r, i j}+\mathbf{M}_{n, i j}\right)
$$

where $\mathbf{v}_{\mathrm{i}}$ and $\boldsymbol{\omega}_{\mathrm{i}}$ are the translational and angular velocities of the particle, respectively, and $\mathrm{k}_{\mathrm{c}}$ is the number of particles in interaction with the particle. As plotted in Fig.1, the forces involved are: the gravitational force $\mathrm{m}_{\mathrm{i}} \mathrm{g}$, and inter-particle forces between particles, which include elastic force $\mathbf{f}_{\mathrm{c}, \mathrm{ij}}$, and viscous damping force $\mathbf{f}_{\mathrm{d}, \mathrm{ij}}$. For fine particles, the van der Waals force $\mathbf{f}_{\mathrm{v}, \mathrm{ij}}$ needs to be considered. These inter-particle forces can be resolved

* Corresponding author: zongyan.zhou@monash.edu 
into the normal and tangential components at a contact point. The torque acting on particle $\mathrm{i}$ by particle $\mathrm{j}$ includes two components: $\mathbf{M}_{\mathrm{t}, \mathrm{ij}}$ which is generated by the tangential force and causes particle $\mathrm{i}$ to rotate, and $\mathbf{M}_{\mathrm{r}, \mathrm{ij}}$, commonly known as the rolling friction torque, is generated by asymmetric normal forces and slows down the relative rotation between particles. For ellipsoids, addition torque $\mathbf{M}_{n, \mathrm{ij}}$ should be added because the normal contact force and van der Waals force do not necessarily pass through the particle centre. Other critical features for ellipsoids are introduced briefly below:

- Determining the interaction forces by calculating the radius $\mathrm{R}^{*}$, which is strongly related to the radii of the curvature at a contact point;

- Using geometric potential algorithm to detect the contacts between ellipsoids; and

- $\quad$ Particle orientation is defined by three Euler angles $(\phi, \Theta, \Psi)$ based on the quaternion method.

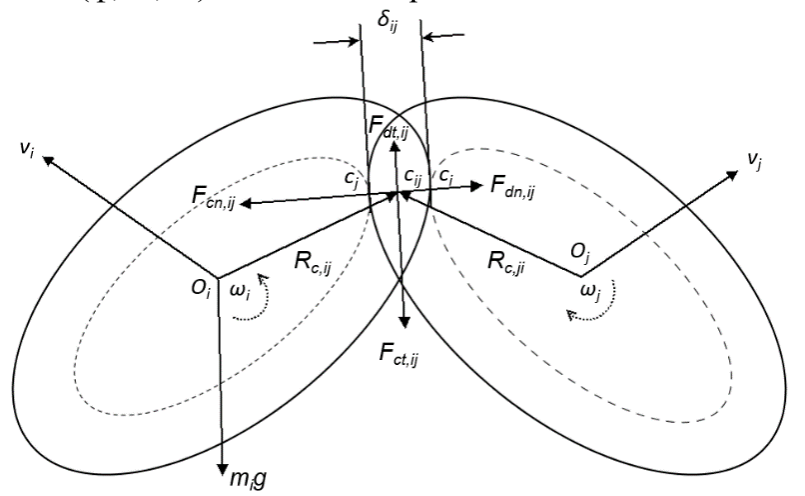

Fig.1. Two-dimensional diagram of forces acting on particle i contacting particles $\mathrm{j}$

For the shape of ellipsoids, three principle diameters are used to describe: $2 \mathrm{a}, 2 \mathrm{~b}$ and $2 \mathrm{c}$, where a is the principal radius in the polar direction, $\mathrm{b}$ and $\mathrm{c}$ are principal radii in the equatorial plane. For spheroids used in this work, $b=$ $\mathrm{c}$ which means that the equatorial plane is a circle. The parameter aspect ratio $A R$ is defined as $A R=a / c$. Spheroids with aspect ratios varying from 0.25 to 3.0 are used in this work. For a prolate $(\mathrm{a}>\mathrm{b}=\mathrm{c})$ spheroid, aspect ratio $A R>1$; for a sphere $(a=b=c), A R=1$; and for an oblate $(\mathrm{a}<\mathrm{b}=\mathrm{c})$ spheroid, $\mathrm{AR}<1$.

In the current study, we quantify the extent of mixing by Lacey mixing index $\mathrm{M}$ given by [9]

$$
M=\frac{\delta_{0}^{2}-\delta_{t}^{2}}{\delta_{0}^{2}-\delta_{r}^{2}}
$$

where $\delta_{t}^{2}$ is the variance of the current state of mixing. $\delta_{0}^{2}=q(1-q)$ and $\delta_{r}^{2}=q(1-q) / p$ are the variances of the in a completely separated system and in a well-mixed system, respectively. $q$ is the total proportion of either type of particles in the mixture. $p$ is the average number of particles in a cell. The size of cells are set as $4 d_{v}$ in the current study, where $d_{v}$ denotes the volume-equivalent diameter of the particles. The sliding friction coefficient of the end-walls are set as 0.04 in the simulation, which is small enough to ignore the effects of end-walls.

\section{Results and discussion}

\subsection{Mixing patterns}

As shown in Fig.2, different mixing patterns can be observed for ellipsoids and spheres. Evidently, the beds of ellipsoidal particles have reached cascading regime, which is characterised by the S-shaped surface. However, the flow surface for spheres remains flat, which means spheres are still in rolling regime. As the drum rotates, more avalanching occurs, and better mixing quality is achieved. Each time when red particles finish flowing downward the free surface, they will re-enter the particle mixture, move upward against the side wall, and will experience the avalanching of the next round. As the bed is turned over repeatedly, more red particles accumulate in the central part of the bed in a spiral shape and less red particles would take part in the avalanching motion. As a result, the bed become more hierarchical, indicating the progress of mixing. After rotating for 6 seconds, obvious stratification structures and large mixing interface can be observed for the beds of ellipsoids, while preliminary state of mixing for spherical particles. At the 8th second, red ellipsoidal particles have distributed in the bed homogeneously with the disappearance of the stratification structure, which indicates a perfectly mixed state of the bed. However, for spheres, stratification structure just appears. From the evolution of the mixing patterns of spheres and ellipsoids, ellipsoids are evidently mixed faster than spheres at the same rotating speed.

\subsection{Effects of aspect ratio on mixing rate}

Figs. 3 and 4 show the temporal evolutions of mixing index for oblate and prolate spheroids. The particles of different colours are completely separated initially, i.e. the mixing index is close to 0 . After sufficient revolution of the drum, both spheres and ellipsoids are able to reach a well-mixed state eventually, i.e. the mixing index is close to 1 . For spheres, the increase of mixing index can be fitted by an error function, which is given by

$$
M(t)=M_{\infty}+\left(M_{0}-M_{\infty}\right) \operatorname{erf}(R t)
$$

Where the error function $\operatorname{erf}(x)=2 / \sqrt{\pi} \int_{0}^{x} e^{-x^{2}} d x$. $M_{\infty}, M_{0}$ and $R$ are the final mixing index, initial mixing index and mixing rate respectively, which can be obtained from the fitting of simulation data. Mixing rate quantifies the speed of mixing of particles in different colours. Obviously, the same fitting function can be applied to the temporal growth of the mixing index for ellipsoids. Moreover, for both oblate and prolate spheroids, the mixing index become steady and close to 1.0 after rotating for around 10 seconds, while 30 seconds for spherical particles. Clearly, ellipsoids are mixed faster than spheres at the same rotating speed. For oblate spheroids, the speed of mixing is similar. The particles with aspect ratio of 0.50 are mixed faster than the ones with aspect ratio of 0.25 . Similarly, slight difference of mixing speed is observed among prolate spheroids of different aspect ratio. The particles with aspect ratio of 2.0 are mixed fastest for prolate spheroids. 

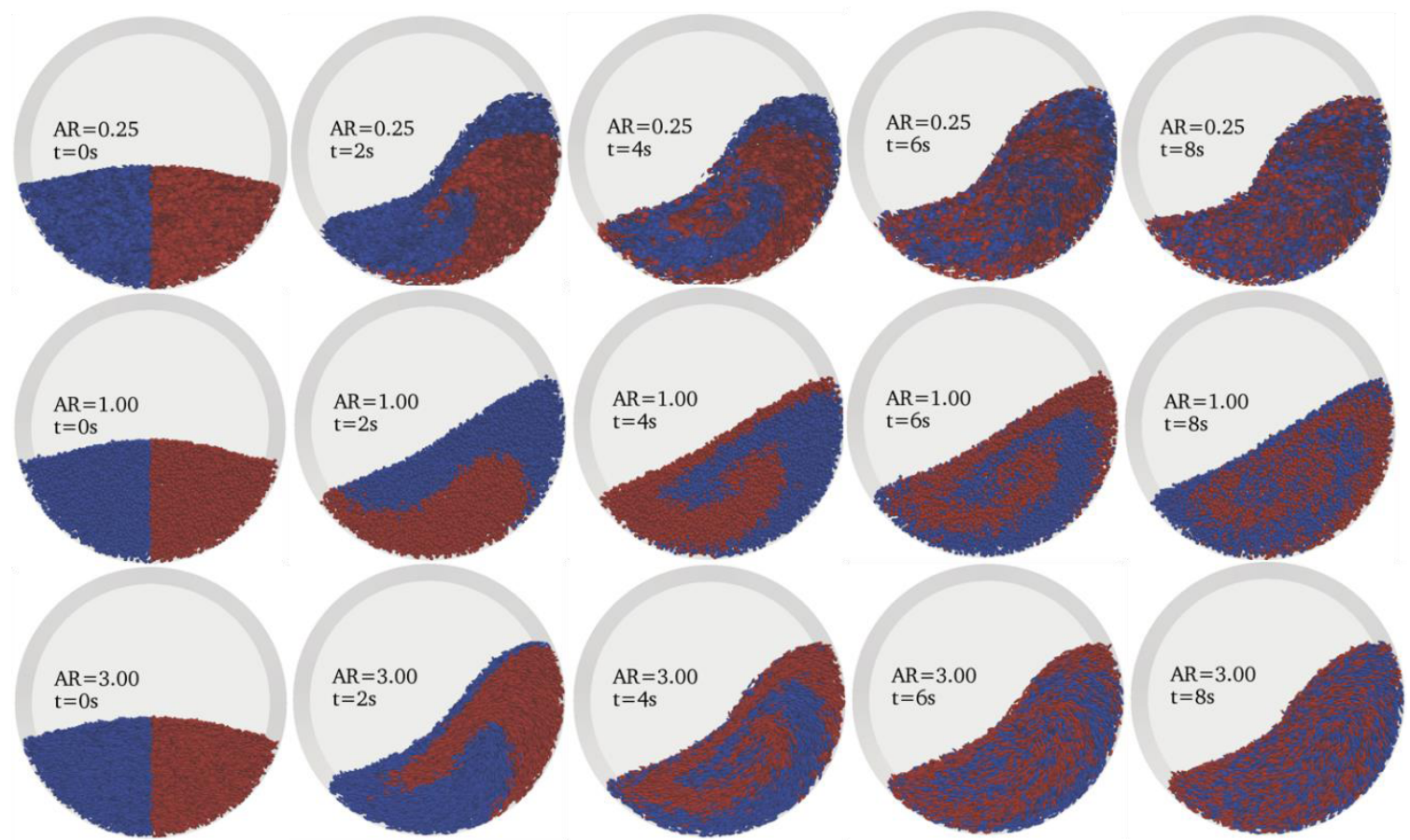

Fig.2. Temporal evolutions of mixing patterns for particles of different aspect ratios at $15 \mathrm{rpm}$.

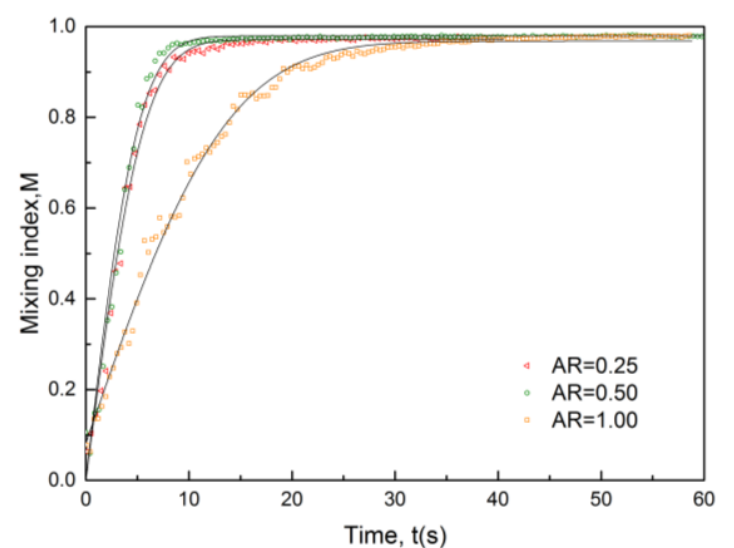

Fig. 3. Evolution of the mixing index of spheres and oblate spheroids with time at $30 \mathrm{rpm}$. The solid curves are the fitting

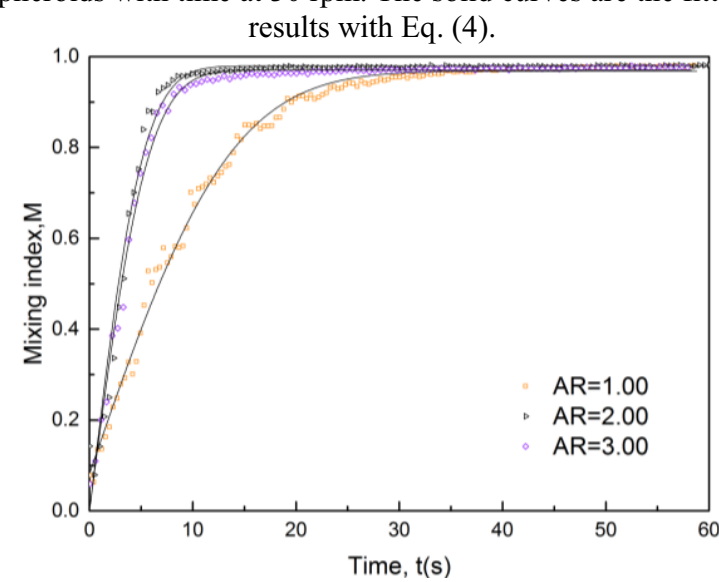

Fig. 4. Evolution of the mixing index of spheres and prolate spheroids with time at $30 \mathrm{rpm}$. The solid curves are the fitting results with Eq. (4).

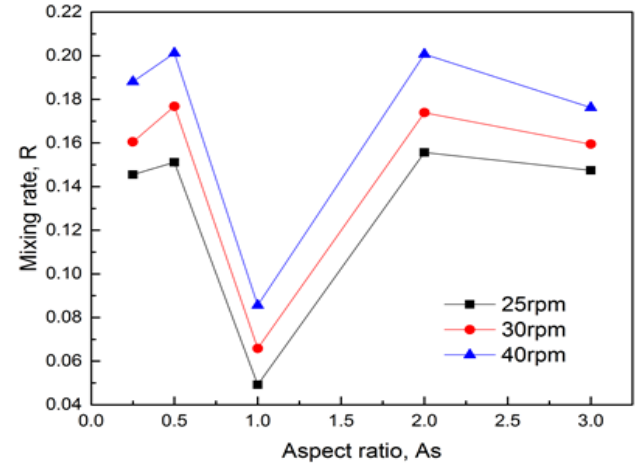

Fig.5. The relationship between mixing rate and aspect ratio for different rotating speeds.

Fig. 5 shows the relationship between the mixing rate and aspect ratio under different rotating speeds. Obviously, for the same rotating speed, spheres are mixed much slower than ellipsoids, which is in consistent with the evolution of mixing patterns. However, for oblate or prolate spheroids, slight change on mixing rate can be observed when the aspect ratio varies. Prolate and oblate spheroids tend to have similar mixing rate when there is similar deviation of the aspect ratio from the unity. Though slight difference of mixing rate is observed between ellipsoidal particles, granules with aspect ratio of 0.50 and 2.0 have the largest mixing rate, which is approximately 3 times faster than spherical particles.

Additionally, when the rotating speed increases from $25 \mathrm{rpm}$ to $40 \mathrm{rpm}$, the mixing rate increases for both spheres and ellipsoids. It is different from the results obtained by Liu et al. [3], where the mixing rate of spheres decreases when the rotating speed increases. It is because the mixing rate obtained in the current study is calculated by the evolution of mixing index with time other than revolutions. It means that, though the mixing efficiency 
for each revolution decreases with the increasing rotational speed [3], the mixing rate increases due to more revolutions are completed in a certain period of time.

\subsection{Evolution of the mixing index in a wide range of rotating speed}

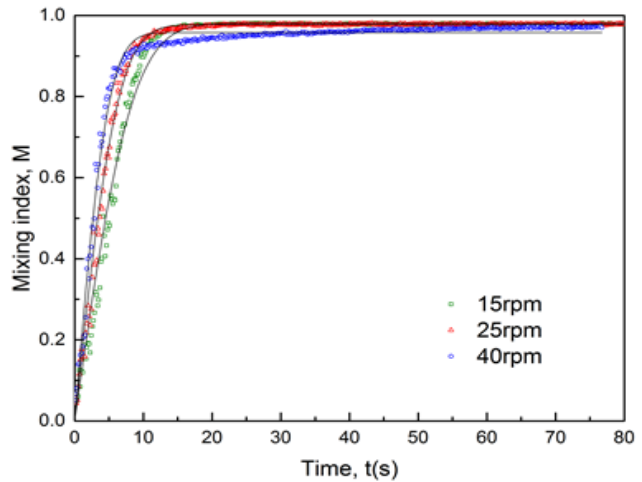

(a)

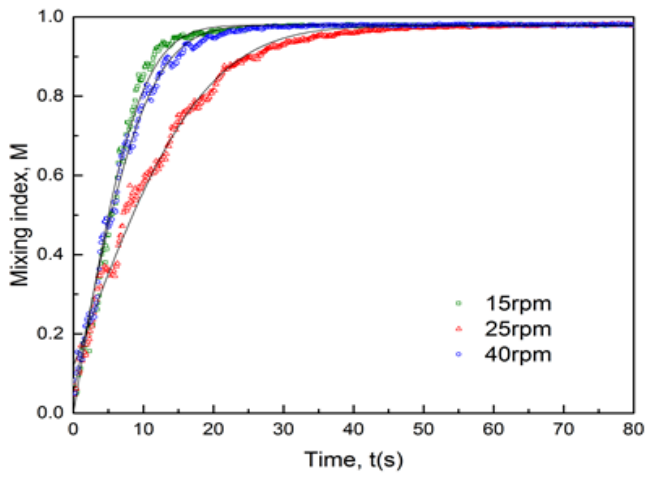

(b)

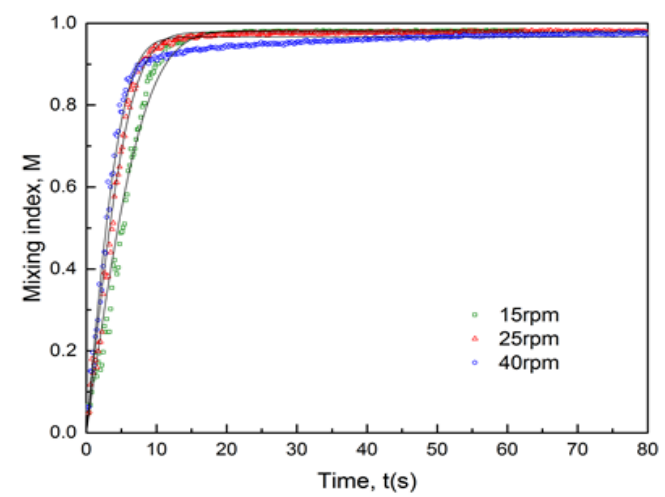

(c)

Fig.6. The temporal variation of mixing index for different rotating speeds: (a) $\mathrm{AR}=0.25$; (b) $\mathrm{AR}=1.00$; (c) $\mathrm{AR}=3.00$.

Fig. 6 shows the temporal evolutions of mixing index of ellipsoids and spheres for different $\mathrm{rpm}$. The inclination of the curve denotes the speed of mixing. Though particle shape changes, an exponential increase of mixing index remains for ellipsoidal particles. For spheres, the speed of mixing is sensitive to the variation of rotational speed. From $15 \mathrm{rpm}$ to $25 \mathrm{rpm}$, the speed of mixing decreases sharply. It means that increasing rotating speed leads to a significant decrease in "mixing efficiency", i.e., the number of revolutions required to turn over the granular bed, which is consistent with the previous study [10]. However, when the rotating speed increases from $25 \mathrm{rpm}$ to $40 \mathrm{rpm}$, the mixing speed increases, which means the mixing speed is dominated by the rotating speed other than the "mixing efficiency".

However, this trend cannot be applied to ellipsoidal particles. Slight change is observed between ellipsoidal particles when rotating speed varies from $15 \mathrm{rpm}$ to 40 $\mathrm{rpm}$. As the rotating speed increases, the mixing speed of ellipsoids increases as well. It means that, with the increasing rotational speed, the "mixing efficiency" does not change much so that the number of revolutions in a certain time dominates the speed of mixing.

\section{Conclusions}

DEM is used to examine the effect of particle shape on the mixing of particles in a rotating drum. It indicates that after sufficient time of revolution, both ellipsoids and spheres can reach well-mixed state. Similar to spherical particles, the mixing index of ellipsoidal particles increases exponentially with time. From $25 \mathrm{rpm}$ to 40 rpm, ellipsoids tend to have larger mixing rate than spheres at the same rotating speed. Though slight difference of mixing rate is observed between ellipsoidal particles, granules with aspect ratio of 0.50 and 2.00 are mixed fastest. For a wider range of rotational speed, i.e. from $15 \mathrm{rpm}$ to $40 \mathrm{rpm}$, the speed of mixing decreases first and reaches a minimum at $25 \mathrm{rpm}$, then increases in the range of $25 \mathrm{rpm}$ to $40 \mathrm{rpm}$. However, for ellipsoidal particles, monotonous increase of mixing speed is observed.

\section{Acknowledgements}

The authors are grateful to Australian Research Council and BlueScope Steel for their financial support of this work.

\section{References}

1. H. Henein, J.K. Brimacombe, A.P. Watkinson, Metall. Trans. B 16, 4 (1985)

2. E. Alizadeh, O. Dubé, F. Betrand, J. Chaouki, AIChe J. 59, 6 (2013)

3. P.Y. Liu, R.Y. Yang, A.B. Yu, Chem. Eng. Sci. 86 (2013)

4. M.M.H.D. Arntz, W.K. den Otter, W.J. Briels, P.J.T. Bussmann, H.H. Beeftink, AIChe J. 54, 12 (2008)

5. F. Geng, Z.L. Yuan, Y.M. Yan, D.S. Luo, H.S Wang, B. Li, D.Y. Xu, Powder Technol. 193, 1 (2009)

6. G.G. Pereira, S. Pucilowski, K. Liffman, P.W. Cleary, Appl Math Model 35, 4 (2011)

7. P.A. Cundall, O.D.L. Strack, Geotechnique 29, 1 (1979)

8. A. Dziugys, B. Peters, Granul Matter 3, 4 (2001)

9. P.M.C. Lacey, J. Appl. Chem. 4, 5 (1954)

10. G.J. Finnie, N.P. Kruyt, M. Ye, C. Zeilstra, J.A.M.Kuipers, Chem. Eng. Sci. 60, 15 (2005) 